\title{
Smelling through microfluidic olfaction technology
}

\author{
Pouria Mehrabi, Justin Hui, Mahyar Mohaghegh Montazeri, Kim Tien Nguyen, Abigail Logel, Allen O’Brien, \\ Mina Hoorfar \\ School of Engineering \\ University of British Columbia \\ Kelowna, Canada \\ mina.hoorfar@ubc.ca
}

\begin{abstract}
Detection of volatile organic compounds (VOCs) in the exhaled breath is found to be a promising method to diagnose different diseases. The amount of alcohol or drugs absorbed/inhaled in the body can also be measured using gas sensors. Oral habits can affect the composition and also concentration of VOCs produced as a result of cellular metabolic reactions inside our body. Recognition of exhaled breath patterns including composition and concentration of VOCs provides useful information regarding how the breath affects the artificial olfaction systems. This can provide a powerful tool to calibrate gas sensors and detect VOCs associated with different diseases.

In the following study, the breath signatures are extracted after different activities including fasting, brushing teeth, and drinking coffee. The results are normalized and implemented into a feature extraction model that extracts principal features for each regime. This will determine the breath signature of each regime. The results show that the effect of these activities on the breath is consistent between different subjects. This study provides the base signature of the exhaled breath which can be used in a clinical setting to identify other target VOCs that are considered the biomarkers of diseases.
\end{abstract}

Keywords - breath analyzer, gas sensor, microfluidic artificial olfaction

\section{INTRODUCTION}

Breath analyzers have been considered as a non-invasive method for detection of chemicals or mixtures of chemicals that are either produced as part of biological metabolism or are absorbed from exogenous sources [1]. The exhaled breath can contain volatile organic compounds (VOCs) (e.g., acetone, ethanol, and isoprene), inorganic compounds (e.g., carbon dioxide and nitrogen), and non-volatile substances (e.g., isoprostanes and peroxynitrite) [2]. The "smell-print" can be used as an indicator of the patient physical condition and lifestyle. Despite its great potential, breath analysis still has several practical challenges. A mixture of inorganic gases (e.g. $\mathrm{CO}_{2}$, ethanol, and $\mathrm{N}_{2}$ ) and VOCs in the exhaled breath of a normal healthy person can be identified (many of these chemicals are absorbed from environment such as smoking, food consumption, bacterial microflora, work environments and medication [3]). However, The exact composition is influenced by diet, age, body mass index (BMI), and gender, and hence, varies between individuals [4]. Although the majority of VOCs in exhaled breath have external sources, they still should be distinguished from the endogenous species.

Another challenge associate with breath analysis is calibration of the sensor based on standard samples. For example, for detection of the cannabis use, it is necessary to control temperature and humidity, and breath samples must be collected from the lower airways (alveolar air). Also, in order to quantify impairment, one must discriminate between THC (the psychoactive component) and CBD.

Finally, identifying a particular health condition is not achievable by detection of a single target gas and instead requires detection of a pattern of compounds [5]. For example, monitoring the level of methane and hydrogen is a promising technique for diagnosis of Small intestinal bacterial overgrowth (SIBO), Irritable bowel syndrome (IBS), and diabetes [6]. However, these gases could be produced as a result of normal digestion and respiration. Therefore, the rate and concentration of these species in exhaled breath should be determined base on each metabolic reaction [7]. Also, malabsorption of lactose and glucose causes huge production of hydrogen and methane in breath [8]. Discovery of the breath pattern as a result of this process will prevent mixing up this malfunction of metabolic system with another disease like diabetes at first stages [9].

The technology presented here has high sensitivity and selectivity, and it uses a single sensor embedded in a microfluidic channel [10]. The latter adds several benefits (such as less calibration complexity, smaller size, and lower cost) compared to traditional e-noses [11]. This technology is used to detect VOCs in the exhaled breath of several subjects after different activities. The purpose of this study is to develop base 
signatures of the breath that can be considered while it is used for detection of a certain biomarker for a disease.

\section{EXPERIMENTAL}

\section{A. Device description}

This technology is portable and easy to assemble, making it ideal for a hand-held device (see Fig. 1). The air chamber is built into the main cartridge, eliminating the need for a separate chamber to be filled with the sample. Both humidity filters (i.e., potassium hydroxide and zeolite) can be easily installed/replaced simply by sliding each piece into the cartridge. A total of three pieces are needed to complete the two filters. The last piece of the filter design includes slots for three springs to be inserted. These are used to make a one-way valve. A rubber stopper is attached to the end of the three springs to prevent a backflow of the air sample. When the breath is blown into the device, the force of the air will compress the springs allowing the air to flow pass the filters and into the sensing chamber. The one way valve housing is bolted to the cartridge to ensure an air right seal and easy assembly. The opening at the end of the one way valve housing allows a disposable mouthpiece to be attached for each new sample.

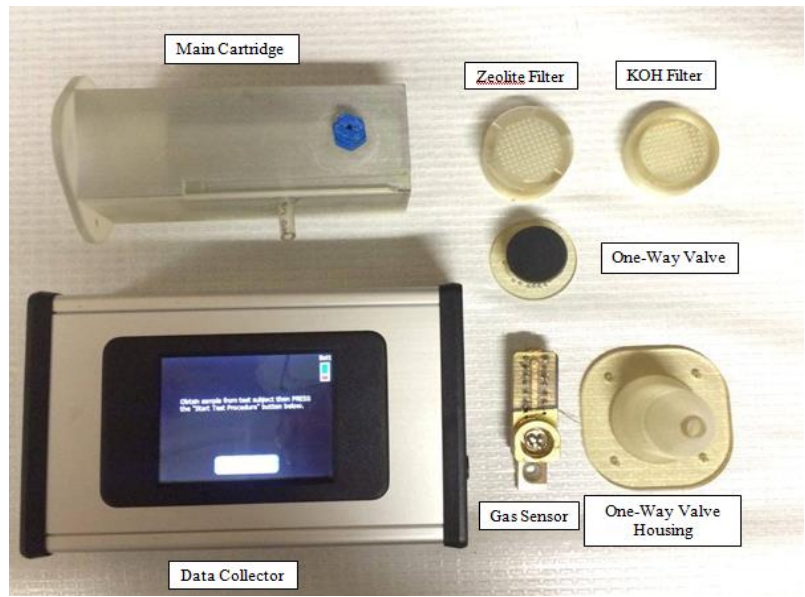

Fig. 1 - Layout of the breathalyzer including each component

A box containing the sensor and electronics is connected to the cartridge and sensing chamber with a push valve. A large bracket will then slide into place following guides imbedded into the cartridge. It can hold the sensing device firmly in place. A schematic of the device including all components and also the test setup is shown in Fig. 2.

\section{B. Materials}

The entire device is 3D printed to reduce machining costs. The cartridge, filters, a one-way valve housing are made of veroclear (UBCO Star 3D printing facility) to allow intricate geometries. Before testing, the cartridge and all of the parts are coated with Parylene $\mathrm{C}$ to prevent diffusion of the gases into the plastic.



Fig. 2 - Schematic of the device and test setup

\section{Testing procedure}

Over the period of testing, the subjects started the experiment by fasting for 12 hours. Following the collection of the breath after fasting, the experiment was carried out by collecting the breath samples after certain activities including drinking black coffee, brushing the teeth (Colgate cavity protection winter fresh), and washing using a mouthwash (Listerine original). After performing each activity, the subject blows into the device twice, giving two samples to ensure the results reproducibility.

\section{RESULTS}

Fig. 3 shows the sensor response (referred to as the "smell print") after each activity for each subject. For every subject, the response was the strongest after using mouthwash. The second strongest response curve is after fasting for 12 hours, which is followed by the signal of the breath sample collected after drinking coffee and finally brushing the teeth. The sensor's strong response after the use of mouthwash is due to the presence of various alcohols (26.9\%) and also organic compounds (e.g. Thymol and Menthol) in the mouthwash solution. Also, since the concentration of ethanol and acetone in the exhaled breath of a person after fasting for 12 hours increases [12], the sensor's second largest response is for the samples collected after fasting. It is worth to mention that regardless of the subject the trend of the response curves associated with each activity is similar. This proves that the "smell print" of each activity is unique in the breath.

The pattern of breath signature across different activities looks similar for that particular activity between different subjects. This similarity can be observed in the slopes of the curve during the rising period (i.e., for the exposure process) and decaying part (i.e., for the recovery stage).

Fig. shows the results of the feature extraction method (performed based on three features including: the area under each curve, the maximum response, and response at $t=200 \mathrm{~s}$ for the breath samples collected after using the mouthwash. The results show that each subject has a unique breath signature after rinsing the mouthwash. Also, the two replications conducted for the breath of each subject are in agreement in 3dimensional space, showing reproducibility of the results. 

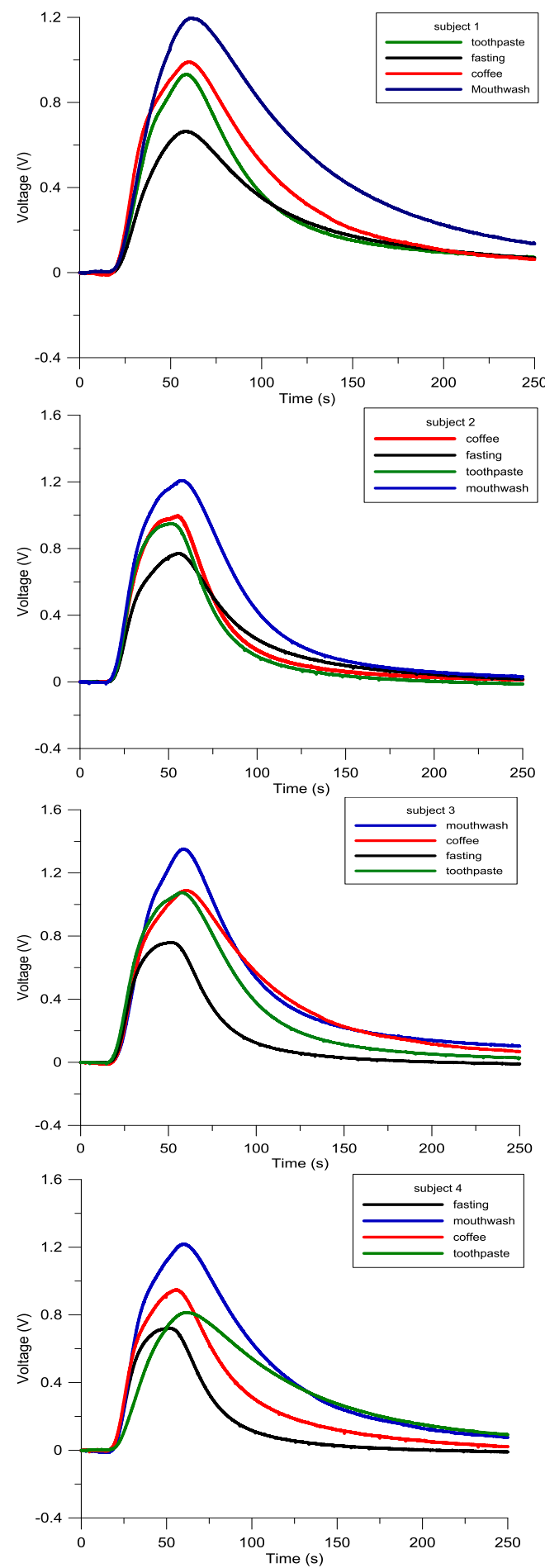

Fig. 3 - Results of the experiment for each subject. The trend in each graph for each activity is similar for different subjects.

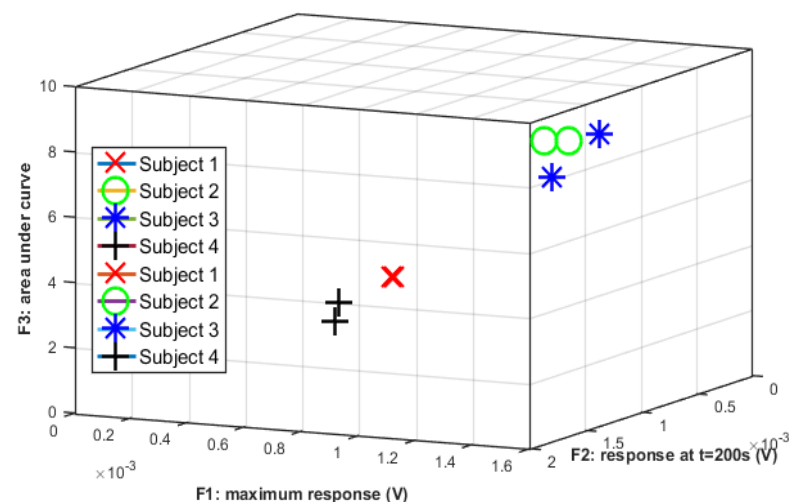

Fig. 4- Feature extraction of results for the breath samples collected after using the mouthwash. These features are based on the area underneath the curve, the maximum response, and response at $\mathrm{t}=200 \mathrm{~s}$.

\section{CONCLUSION}

The response of the microfluidic olfaction breath analyzer showed that the trend for the breath samples collected after different activities is consistent regardless of the subject. The response of the sensor ("smell print") is much stronger after the use of mouthwash and fasting due to high amount of alcohol existing in exhaled breath in both of these activities. The experimental results were reproducible for each subject as the signal magnitude trend between different activities was the same in all cases. For the future study, a diverse range of subjects will be tested to observe and extract biomarker patterns in a wide variety of cases.

\section{AKNOWLEDGMENT}

We would like acknowledge the Natural Sciences and Engineering Research Council of Canada (NSERC) for funding this research.

\section{REFERENCES}

[1] K.-H. Kim, S. A. Jahan, and E. Kabir, "A review of breath analysis for diagnosis of human health," TrAC Trends Anal. Chem., vol. 33, pp. 1-8, Mar. 2012

[2] P. Kintz, P. Mura, C. Jamey, and J.-S. Raul, "Detection of $\Delta 9$ tetrahydrocannabinol in exhaled breath after cannabis smoking and comparison with oral fluid," Forensic Toxicol., vol. 35, no. 1, pp. 173178, Jan. 2017.

[3] L. Blanchet et al., "Factors that influence the volatile organic compound content in human breath,” J. Breath Res., Jan. 2017.

[4] C. Di Natale, R. Paolesse, E. Martinelli, and R. Capuano, "Solid-state gas sensors for breath analysis: A review," Anal. Chim. Acta, vol. 824, pp. 117, May 2014.

[5] K. Arshak, E. Moore, G.M. Lyons, J. Harris, and S. Clifford, "A review of gas sensors employed in electronic nose applications," Sens. Rev., vol. 24, no. 2, pp. 181-198, Jun. 2004. 
[6] Ledochowski M, Sperner-Unterweger B, Widner B and Fuchs D 1998 Fructose malabsorption is associated with early signs of mental depression Eur. J. Med. Res. 3 295-8

[7] de Lacy Costello, B. P. J., M. Ledochowski, and Norman M. Ratcliffe. "The importance of methane breath testing: a review." Journal of breath research7.2 (2013): 024001.

[8] Ruzsanyi, Veronika, et al. "Diagnosing lactose malabsorption in children: difficulties in interpreting hydrogen breath test results." Journal of breath research 10.1 (2016): 016015.

[9] Eisenmann A, Amann A, Said M, Datta B and Ledochowski M 2008 Implementation and interpretation of hydrogen breath tests J. Breath Res. 2046002
[10] Mohammad Paknahad, Jannat Singh Bachhal, Ali Ahmadi, Mina Hoorfar, "Highly selective multi-target 3D-printed microfluidic-based breath analyzer" Micro Electro Mechanical Systems (MEMS), 2016 IEEE, (2016) 905-908

[11] Alphus D. Wilson, and Manuela Baietto, "Advances in Electronic-Nose Technologies Developed for Biomedical Applications”, Sensors 2011, 11, 1105-1176.

[12] Kalapos, M. P. "Possible physiological roles of acetone metabolism in humans." Medical hypotheses 53.3 (1999): 236-242. 